\section{PUERPERAL ALBUMINURIA.}

\section{To the Editors of THE LANCET.}

SIRS,-Dr. Barnes, in your issue of May 12th, has suggested that a study of the phenomena of gestation may throw light upon certain pathological conditions. As regards the subject of his article, I think the converse is also true. In The Lancex for 1886,1 pointed out various similarities between puerperal albuminuria and some forms of functional albuminuria, and suggested that the puerperal disorder was due primarily to a blood change and was independent of organic renal disease. There is one form of functional albuminuria of which the pathology is fairly well known, and if a comparison is made between cases of this disorder and others of puerperal albuminuria the correct pathology of the latter will be clear.

Patients not unfrequently present themselves to the physician complaining of a complexity of symptoms, of which the chief are languor, mental torpor, sleeplessness and yet drowsiness, and a throbbing headache often felt at the back of the head. The pulse is found to be one of high arterial tension, and if the patient is beyond middle age some enlargement of the heart may be discovered. With these conditions the urine may or may not contain albumen. The history will often show that the patient has been in the habit of eating and drinking too much or of taking insufficient exercise, although in other cases none of these causes are found, and it would then appear that there is some constitutional tendency to the condition. The pathology of such cases is the following: From excessive imports or diminished exports the blood is loaded with impurities causing the high arterial tension and the enlargement of the heart, which may be due to dilatation or to hypertrophy according to the duration of the disorder. The kidneys, in their efforts to get rid of the blood impurities, are liable to become overworked and irritated, and albuminuria ensues. The symptoms are relieved by a dose of calomel, followed by a saline purge; the high arterial tension is diminished, and the albuminuria vanishes at once, the explanation being that the purge has removed the blood poison from the system, and with it the source of irritation of the kidneys. The albuminuria is clearly functional, and its features are strikingly similar to those of puerperal albuminuria.

In pregnancy we find, as a rule, high arterial tension and some enlargement of the heart, both conditions being the more marked the later the period of gestation. The vascular system of the mother alone bas to envey the excrementitious matters of both mother and child. The blood is therefore impure, as in the functional albuminurias described above, and in its circulation causes high arterial tension and cardiac strain. The kidneys endeavour to remove the impurities, and in most cases are successful. In other cases, whether from excessive amount of impurities or from excessive vulnerability of the kidneys, these organs are irritated by their efforts, and albuminuria ensues.

But the analogy between the two conditions mentioned does not cease here. In the one case the dose of calomel removes some of the blood impurities, and with them the albuminuria and other symptoms. In the albuminuria of pregnancy parturition plays the same rôte by removing the child, and with it the extra source of blood impurity ; consequently the high arterial tension and albuminuria at once disappear. An albuminuria consequent upon organic disease of the kidneys could not disappear so quickly, never to return, and we are forced to believe that the pathology in the two classes of cases is the same, and that the albuminuria of pregnancy is primarily functional. This view is supported by the observations of Depaul, mentioned by Dr. Barnes. It is nevertheless true that in post-mortem examinations upon cases of puerperal albuminuria organic disease of the kidneys has been'found. In very few instances has venous congestion been seen. Usually, if changes are present, they are described as those of a more or less intense parenchymatous nephritis. I have elsewhere shown that such changes are found in connexion with the albuminuria of diabetic coma, where they are probably produced by an attempt on the part of the kidneys to remove bodies of the acetone group from the system. It would be interesting to know whether Weigert's coagulation necrosis, which is such a marked appearance in the acetonæmic kidney, and would indicate intense irritation, is also present in the kidney of puerperal albuminuria. On this point I have no information to give, as I have not yet had an opportunity of examining the kidney in such a condition. The finding of organic change of any kind would not invalidate the view that puerperal albuminuria is usually functional.

The degree of puerperal blood impurity varies, and may in one case produce merely high arterial tension; in another also a slight albuminuria; in a third, assisted possibly by an unusual vulnerability of the kidney, a severe albuminuria and organic renal change. It will be remembered that the functional albuminurias referred to above, not of puerperal origin, do sometimes lapse into organic disease. Here, however, the irritation being more persistent, a granular kidney is produced instead of a more acute nephritis.

I am, Sirs, yours obediently,

Robert MagUire, M.D., M.R.C.P., Seymour-street, Portman-square, W., May 12th, 1888.

\section{"PHTHISIS A DISEASE OF THE NIGHT."} To the Editors of THE LANCET.

SIR's,-I have to thank Dr. Richardson for his kindly criticism, but I think that the valuable facts he adduces from Dr. Buchanan's report only serve to corroborate my theories. I learn from them that the effects of cold air breathed at night are greatly lessened when the amount of watery vapour in the air is permanently reduced. Dr. Huggard of Davos, too, informs me that the atmosphere there is singularly free from watery vapour, and on that account the cold, which is intense, is hardly noticeable. It is a fair question to ask, in view of Dr. Ransom's very able and interesting address-What is cold? I suppose that it is a mere negation, not an entity. It is certain, I think, that these bodies of ours do resent in many and varied ways the abstraction of even small amounts of the heat which they must maintain, or die. It seems almost a pity that cold cannot be looked upon as an entity, for it is certain that the effect of the want of heat or of its abstraction differs greatly under varying circumstances. We speak of cold and damp as if cold were one thing and damp another. In point of fact, cold is simply want of heat, a mere nonentity, whlle damp (vapour of water) is a very distinct entity. Cold affords (if I have read Dr. Ransom's most valuable paper aright) a good example of an "unfixed term." Cold is, however, too handy a term to drop before I have said that its effects seem to vary vastly according to-(1) its degree or intenseness, its kind, its mode of application, and to the duration of the exposure to it, \&c.; (2) to the state aud condition of its subject in whole or in part, and also to the states and conditions in whole or in part of other living organisms with which it may be brought in contactmen, beasts, bacilli, miasmata, \&c.

Space will not allow me to say mora. To repeat the main points of my paper, which might have been headed "No Irritation, no Phthisis," only that I wished to emphasise my opinion that in the vast majority of cases the chief mischief is done at night: 1. Irritation (another unfixed term) is the cause of phthisis. 2. Cold air breathed at night is the commonest form of irritation causing phthisis. 3. Therefore a supply of pure warm air at night would stamp out phthisis. Phthisis is undoubtedly a disease of the so-called temperate climates. There must be some form of irritation common to all to cause the vast majority of cases. Particular forms of irritation, such as grit, coal-dust, \&c., do not cause a different disease. It is an established fact that persons who would otherwise inevitably succumb to the disease can ward it off if only they have the means and will follow out sound directions as to climate and regimen.

Now, I believe, and write to say so, that if only we could render the populations of temperate climates independent of climate by night by supplying them with pure warm air at night, then phthisis would be as unknown as it is in some parts of Upper Egypt, or, to take the other extreme, in Iceland. A man awake can look after himself, asleep ho cannot. During our waking hours it seems clear to me that our well-known five senses, togsther with the sixth or muscular sense, combine so as to form, as it were, a seventh or combined sense, which acts so as to bring about or produce the state or condition which may be termed "consciousness." This enables us to steer our sentient bodies clear of the thousand surrounding points of contact which would other- 